\title{
Research of Wind Power Prediction Based on the Auto-Regressive Model
}

\author{
L. Feng \\ School of Electrical and Information \\ Changchun Institute of Technology Jilin \\ Changchun, China
}

\author{
C.H. Liang \\ School of Electrical and Information \\ Changchun Institute of Technology Jilin \\ Changchun, China
}

\author{
H. Huang \\ Logistics Services Center Jilin Province Electric Power Limited Company \\ Changchun, Jilin, China
}

\begin{abstract}
This paper discusses in detail the reason for the inaccurate result from the present system for wind farm output power prediction. Time series analysis method was applied for improving existing problem in prediction model and treatment method of basic data. Improving self-regressive mathematical model was established and taken the model identification. Using SPSS software simulates and further assists model identification and using given wind farm historical output power data to forecast one and multi-wind power unit output power in oddnumber days and a week. Finally, this paper compares and analyses the getting prediction power and expound the next step work that improves the wind power prediction accuracy.
\end{abstract}

Keywords-wind power prediction; time series analysis; selfregressive mathematical model; simulation

\section{INTRODUCTION}

Wind power technology is current renewable energy utilization of the most mature technology and the most largescale development and commercialization development prospects. With the development of wind power technology development, its malpractice appears gradually. Intermittency and fluctuation of wind power itself unique determines the power of intermittent and fluctuating. In order to satisfy the power supply demand, ensuring the reliability of the stable operation of the power grid and power supply system, so, we need to effectively plan and schedule for the power supply system. While the wind power intermittent itself unique and uncertainty, increase the difficulty of grid scheduling and the reserve power capacity. In order to solve the distribution problem of instability and reduce the power grid reserve capacity cost, we must predict the output power of large-scale wind farms. Through real-time accurate prediction for wind power generation, power dispatching department can advance the sch eduling plan according to the wind power change in order to ensure the power balance and the safety operation of the power grid, and adjust accordingly to effectively mitigate the adverse impact of wind power on power grid and effectively reduce the operation cost and improve the efficiency of wind power generation. Therefore, how to forecast the wind power as accurately as possible is an urgent problem to be solved.

\section{AR MATHEMATICAL MODELING}

We apply the stochastic process theory and mathematical statistical methods to study statistical regularities followed by random data sequence. Random data is arranged based on chronological sequence. It possesses good predictability using time series analysis to analyze smooth time series data. The ARMA model consists of two models of the AR (p) model and the MA (q).

\section{A. ARMA Model Identification Criteria}

If stationary series $\mathrm{x}(\mathrm{t})$ is tailing the autocorrelation function, partial autocorrelation function for the censored sequence, $x(t)$ is the AR sequence. If stationary series $x(t)$ is the autocorrelation function of the truncation, partial autocorrelation function is tailing sequence, then, $x(t)$ is the MA sequence. If the autocorrelation function and partial autocorrelation function of stationary series $\mathrm{x}(\mathrm{t})$ is all tailing, $\mathrm{x}$ (t) is the ARMA series. Where the self-correlation function is $\rho_{k}=r_{k} / r_{0} \quad$ The self-covariance function
$r(k)=\frac{1}{n} \sum_{t}^{n-k}\left(x_{t+k}-\bar{x}\right)\left(x_{t}-\bar{x}\right)$, among, $\bar{x}=\frac{1}{n} \sum_{t=1}^{n} x_{t}$ is the sample mean. Based on model identification criteria, we analyze data and complete the establishment of the mathematical model.

\section{B. Model Identification}

For example, wind power data of certain wind power plant; we adopt historical data to model from May 10 to May 30, 2006 and for the test data on May 31. We use the historical data to draw a line chart from May 10 to May 30, 2006, Figure. I show: 


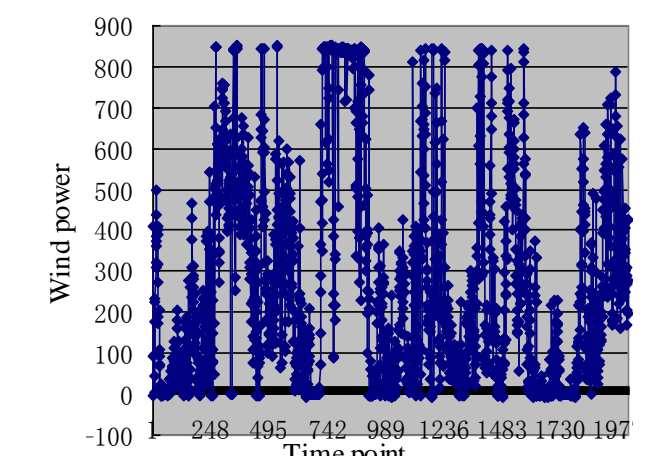

FIGURE I. A WIND FARM WINDP POWER HIST ORY DATA.

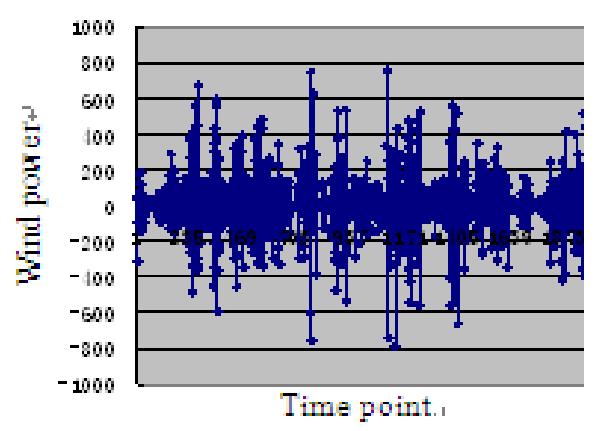

FIGURE II. A FIRST -ORDER DIFF.

As can be seen from the Figure I, the data has large fluctuations and is not stable related time series data, and therefore, we need to make odd transform for data and difference calculation. We take first-order differential operation for the data, the point data shown in figure 2.

By a first-order differential diagram, we can know, the value of wind turbine power is always in the vicinity of a constant random fluctuation, and fluctuations are of the range bounded, no clear trend and cycle characteristics, we regard it as smooth sequence. Therefore, we can further analyze and model based on this data.

\section{AR SimUlation MODELING}

We apply SPSS software to simulate self-correlogram and assist further to make model identification, autocorrelation shown in Figure 3 and Figure 4:

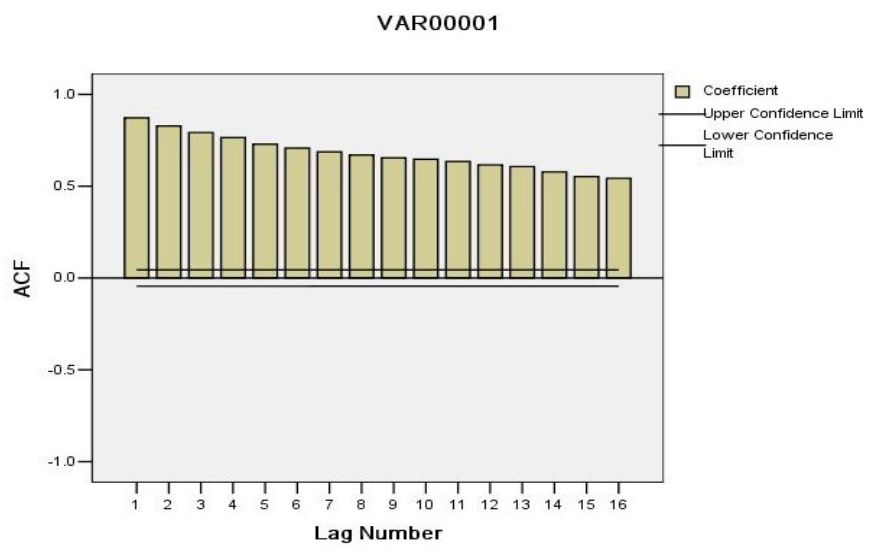

FIGURE III. AR CORRELATION.

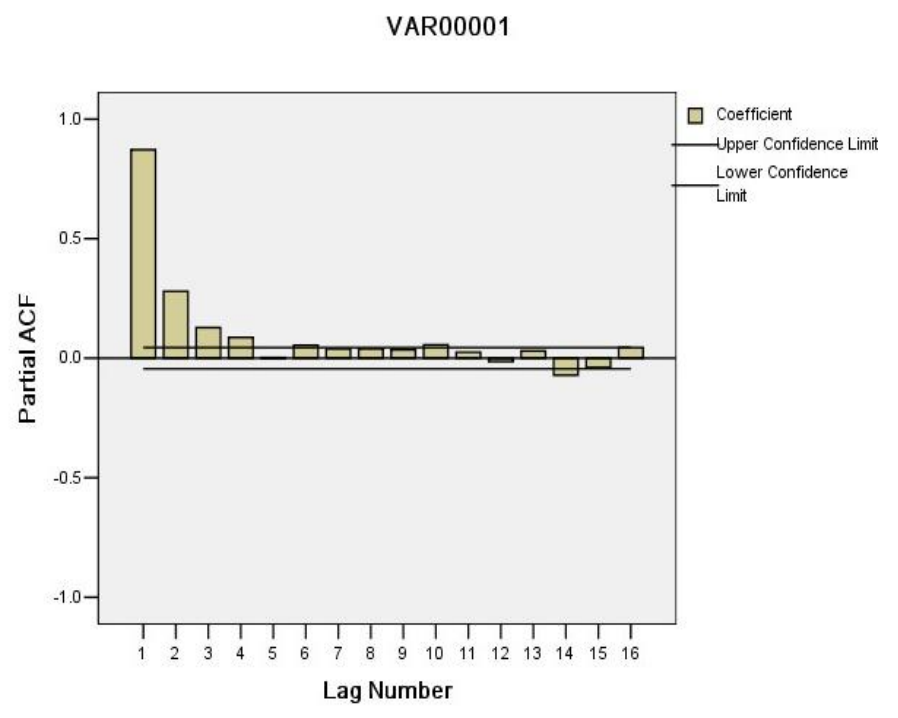

FIGURE IV. MA CORRELATION.

As can be seen from Figure 3, model AR (P) analyzed for auto-correlation function of stationary sequence $\mathrm{x}(\mathrm{t})$ which is derived from data analysis is tailing. The correlation of partial autocorrelation function correlation established by the MA model is censored, namely, AR (P) model accord with time series analysis and establish $\mathrm{AR}(\mathrm{p})$ model, take a $\mathrm{P}=2$.

\section{A. Establishing Autocorrelation Function}

We establish the stationary model

$$
x_{t}=\phi_{1} x_{t-1}+\phi_{2} x_{t-2}+\ldots \ldots+\phi_{p} x_{t-p}+\varepsilon_{t} \text {, and multiply at the }
$$
equal sign by $x_{t-k}, \forall k \geq 1$, and then obtain its expectations, gain the equation:

$$
E\left(x_{t} x_{t-k}\right)=\phi_{1} E\left(x_{t-1} x_{t-k}\right)+\ldots \ldots+\phi_{p} E\left(x_{t-p} x_{t-k}\right)+E\left(\varepsilon_{t} x_{t-k}\right), \forall k \geq 1
$$

According to the conditions of the AR (p) model, we can get $E\left(\varepsilon_{t} x_{t-k}\right)=0, \forall k \geq 1$, so we can get the recurrence formula of self-covariance function:

$$
r_{k}=\phi_{1} r_{k-1}+\phi_{2} r_{k-2}+\ldots \ldots . \phi_{P} r_{k-P}
$$

Because of $\rho_{k}=r_{k} / r_{0}, r_{k}$ is divided by the variance function $r_{0}$, and gain recurrence formula of self-correlation function $\rho_{k}=\phi_{1} \rho_{k-1}+\phi_{2} \rho_{k-2}+\ldots \ldots+\phi_{p} \rho_{k-p}$.

We can calculate on the data basis of the above formula obtained and obtain $\bar{x}, r_{k}, \rho_{k}$, and then $\phi_{1}, \phi_{2}, \ldots \ldots \phi_{p}$.

Using SPSS software analysis, we get simulation results shown in Figure 5: 


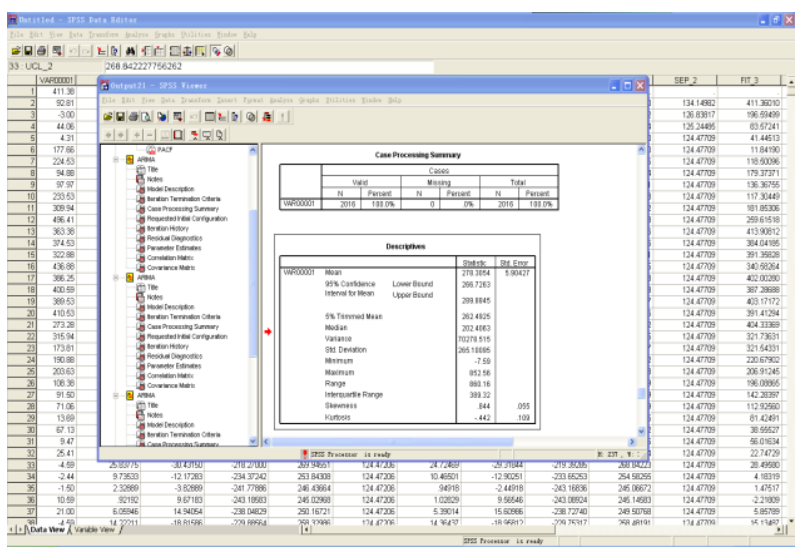

FIGURE V. SPSS SIMULATION RESULT.
From the Figure, we obtain the value of the upper bound 289.8845 and lower bound values 266.7263 at the $95 \%$ confidence interval.

\section{B. Wind Power Prediction of Single-day and One Week}

We use AR (p) to predict 96 point-in-time sequence wind power data for the 1st wind turbines of certain wind farm from 0:00 to 23:45 on May 31, shown in Table 1:

We use AR (p) to gain wind power forecast value and the actual value of 58 wind turbine for certain wind farm, line graph shown in Figure 6:

TABLE I. AR(P) MODEL FOR FORECAST OFWINDPOWERDATA.

\begin{tabular}{|c|c|c|c|c|c|}
\hline Time Point & Power Prediction & Time Point & Power Pre diction & Time Point & Power Pre diction \\
\hline 1 & 214.46 & 33 & 175.2 & 65 & 144.12 \\
\hline 2 & 172.13 & 34 & 333.33 & 66 & 171.19 \\
\hline 3 & 213.76 & 35 & 168.81 & 67 & 184.11 \\
\hline 4 & 263.7 & 36 & 333.33 & 68 & 193.96 \\
\hline 5 & 208.98 & 37 & 175.98 & 69 & 333.33 \\
\hline 6 & 163.43 & 38 & 333.33 & 70 & 333.33 \\
\hline 7 & 206.88 & 39 & 218.65 & 71 & 175.27 \\
\hline 8 & 237.07 & 40 & 148.37 & 72 & 333.33 \\
\hline 9 & 229.88 & 41 & 165.56 & 73 & 333.33 \\
\hline 10 & 204.65 & 42 & 173.9 & 74 & 333.33 \\
\hline 11 & 202.94 & 43 & 146.02 & 75 & 333.33 \\
\hline 12 & 197.02 & 44 & 161.55 & 76 & 172.79 \\
\hline 13 & 204.06 & 45 & 185.88 & 77 & 213.2 \\
\hline 14 & 212.89 & 46 & 174.36 & 78 & 182.47 \\
\hline 15 & 226.14 & 47 & 333.33 & 79 & 183.25 \\
\hline 16 & 196.69 & 48 & 160.34 & 80 & 188.58 \\
\hline 17 & 156.59 & 49 & 333.33 & 81 & 208.69 \\
\hline 18 & 184.32 & 50 & 199.06 & 82 & 219.55 \\
\hline 19 & 185.86 & 51 & 333.33 & 83 & 151.29 \\
\hline 20 & 181.57 & 52 & 333.33 & 84 & 182.79 \\
\hline 21 & 207.74 & 53 & 333.33 & 85 & 333.33 \\
\hline 22 & 197.95 & 54 & 240.67 & 86 & 333.33 \\
\hline 23 & 190.11 & 55 & 226.57 & 87 & 198.43 \\
\hline 24 & 198.56 & 56 & 333.33 & 88 & 333.33 \\
\hline 25 & 139.15 & 57 & 333.33 & 89 & 204.39 \\
\hline 26 & 83.15 & 58 & 263.94 & 90 & 207.71 \\
\hline 27 & 128.06 & 59 & 185.89 & 91 & 333.33 \\
\hline 28 & 92.29 & 60 & 333.33 & 92 & 333.33 \\
\hline 29 & 144.13 & 61 & 138.16 & 93 & 333.33 \\
\hline 30 & 151 & 62 & 216 & 94 & 220.93 \\
\hline 31 & 125.9 & 63 & 152.84 & 95 & 218.81 \\
\hline 32 & 160.9 & 64 & 191.18 & 96 & 181.14 \\
\hline
\end{tabular}

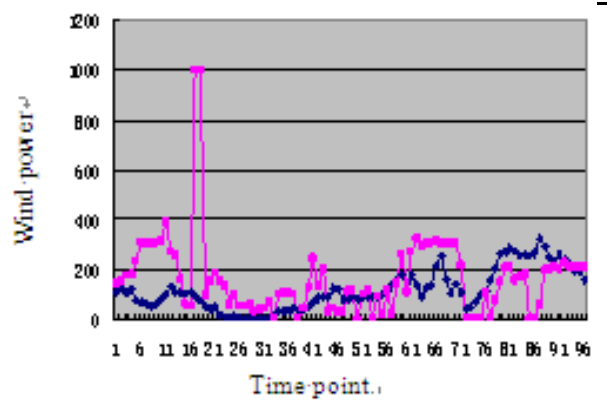

FIGURE VI. COMPARISON OF 58 WIND TURBINE WIND POWER FORECAST VALUE AND THE ACTUAL VALUE.

\section{Simulation MODELING RESUlTs}

According to accuracy rate formu la of the wind farm power forecasting

$$
r_{1}=\left(1-\sqrt{\frac{1}{N} \sum_{k=1}^{N}\left(\frac{P_{M k}-P_{P k}}{C a p}\right)^{2}}\right) \times 100 \%
$$

And the pass rate is calculated

$$
r_{2}=\frac{1}{N} \sum_{k=1}^{N} B_{k} \times 100 \%
$$


Based on prediction results, we analyze comparatively for prediction data and the actual data from the two aspects of accuracy and pass rate, as shown in Table 2 and Table 3:

TABLE II. MAY31ST 00:00 TO23:45 FORECAST ACCURACY.

\begin{tabular}{|c|c|c|}
\hline Wind Turbine & Accuracy & Pass rate \\
\hline No1 & $77.29 \%$ & $86.32 \%$ \\
\hline No2 & $76.34 \%$ & $87.49 \%$ \\
\hline No3 & $75.64 \%$ & $84.53 \%$ \\
\hline No4 & $79.47 \%$ & $82.48 \%$ \\
\hline 4Wind Turbine & $80.42 \%$ & $79.53 \%$ \\
\hline 58Wind Turbine & $80.94 \%$ & $76.48 \%$ \\
\hline
\end{tabular}

TABLE III. MAY 31ST 00:00 TO23:45 IN JUNE 6TH FORECAST ACCURACY.

\begin{tabular}{|c|c|c|}
\hline Wind Turbine & Accuracy & Pass rate \\
\hline No1 & $77.05 \%$ & $81.86 \%$ \\
\hline No2 & $76.55 \%$ & $87.57 \%$ \\
\hline No3 & $74.92 \%$ & $83.82 \%$ \\
\hline No4 & $79.79 \%$ & $81.89 \%$ \\
\hline 4Wind Turbine & $80.32 \%$ & $79.52 \%$ \\
\hline 58 Wind Turbine & $80.14 \%$ & $76.54 \%$ \\
\hline
\end{tabular}

We analyze the forecast data obtained through simulation modeling, the modeling and analysis of time series method is suitable for linear problems. When the prediction step is relatively larger, it reflects good predictability. With the increase of the predicted time, the superiority of the time-series analysis model is increasingly apparent. Moreover, with the increase of the predicted time, the advantage of the ARMA model is gradually increasing and improve more obvious. Therefore, time series method is to be more effective for forecast the output power in the short-term real-time forecast.

\section{ACKNOWLEDGEMENT S}

This work was supported by the Science and Technology development plan project of Department of Science and Technology of Jilin Province (120130169), China.

This work was supported by the Science and Technology Research Project in 12th Five-Year Periods of Department of Education of Jilin Province (2013, 304), China.

\section{REFERENCES}

[1] Xiuyuan Yang, Yang Xiao, and Shuying Chen, "Research of Wind Speed and Wind Power Prediction," Proceedings of the CSEE, 2005, vol. 25 (11): 3, pp.1-5.

[2] Wei Xiong, Operational Research, Beijing: Machinery Industry Press, 2005.

[3] Xingjia Yao, Wind Power Generation Test Technology, Beijing: Electronic Industry Press, 2011.

[4] Guixing Yang, Xiqiang Chang, Weiqing Wang, and Xiuping Yao, "Discussion of the Forecast Precision for Wind Power Forecasting System,"Power System and Clean Energy, 201 1, vol. 27 (1), pp67-71. 